\title{
Une nouvelle Filaire d'Ecureuil en Thailande, Breinlia (B.) manningi n. sp., et son développement chez Aedes (1)
}

\author{
par O. BAIN *, G. PETIT *, N. RATANAWORABHAN **, S. YENBUTRA ** \\ et A.-G. CHABAUD * \\ * Laboratoire de Zoologie (Vers), associé au C.N.R.S., Muséum national \\ d'Histoire naturelle, 43, rue Cuvier, F 75231 Paris Cedex 05. \\ ** Thailand Institute of Scientific and technological Research, \\ 196 Phahonyothin Rd., Bangkhen, Bangkok, Thailand.
}

RESUME. B. (B.) manningi n. sp., parasite du Sciurinae Menetes berdmorei en Thaïlande, est très proche de $B$. (B.) sergenti, parasite de Lémurien d'Asie. Comme chez les autres Breinlia, la larve se développe dans le tissu adipeux de moustiques (Aedes), et la forme infestante, longue d'un peu plus d'1 mm, a une queue proportionnellement très grande $(75-92 \mu \mathrm{m})$, terminée par une pointe conique et deux languettes latérales bien saillantes, pointues ou émoussées.

A new Filaria of a squirrel in Thailand, Breinlia (B.) manningi n. sp., and its development in Aedes.

SUMMARY. B. (B.) manningi, a parasite of Menetes berdmorei (Sciurinae) in Thailand, is very close to B. (B.) sergenti from an Asian Lemur. As in the other Breinlia, the larva develops in adipose tissue of mosquitoes (Aedes) and the infective form, a little longer than $1 \mathrm{~mm}$, has a proportionnally very long tail $(75-92 \mu \mathrm{m})$, ended by a conical tip and two lateral salient more or less conical processes. Santé.

(1) Ce travail a pu être effectué grâce à une subvention de l'Organisation Mondiale de la Accepté le 23 juin 1980. 
Un des 7 Menetes berdmorei (Blyth, 1849) capturés en Thaillande (Kanchanabury), en août 1979, présente une faible microfilarémie (1 microfilaire pour 15 à $20 \mu \mathrm{l}$ de sang). La microfilaire, longue de $235 \mu \mathrm{m}$, large de 5,5 $\mu \mathrm{m}$, à long filament caudal, paraît correspondre à celle trouvée par Manning et coll. (1972) chez le même hôte et dans la même région.

Le développement larvaire est réalisé au laboratoire chez Aedes aegypti et A. togoi.

A la mort du Menetes, les Filaires adultes sont récoltées dans la cavité thoracique. Il s'agit d'un nouveau Breinlia, dont nous donnons la description.

\section{Description des adultes}

MATÉRIEL : 1 holotype, $1 \delta$ allotype coupé en deux, 1 extrémité antérieure de $q$. Les spécimens sont déposés au M.N.H.N., Paris ( $\mathrm{n}^{\circ} 155$ FP).

La morphologie est représentée sur les figures 1 et 2 . Tête brusquement rétrécie. Papilles céphaliques volumineuses avec bouton terminal saillant; papilles labiales externes plus petites. Dans le plan latéral, de la périphérie vers la bouche, on observe les racines nerveuses des amphides, qui, bien que situées en profondeur, sont parfaitement visibles en vue apicale, puis les pores amphidiaux formant deux petites fentes situées sur le cercle des papilles labiales externes, enfin les 2 papilles latérales vestigiales, qui n'atteignent pas la surface de la cuticule et ne sont visibles qu'en vue médiane.

Capsule buccale : un segment antérieur à paroi mince entourant la cavité buccale ovale et comprimée latéralement à ce niveau; un segment postérieur épais et bien cuticularisé entourant la cavité buccale, de section transversale triangulaire.

Microfilaires : pas de gaine; région antérieure aussi large que le corps; crochet exigu ; tête de forme différente suivant son orientation : à apex arrondi quand le crochet est vu de face, à apex oblique quand il est vu de profil ; long filament caudal anucléé.

\section{Dimensions :}

— $q$ holotype : corps long de $53 \mathrm{~mm}$ et large de $310 \mu \mathrm{m}$; anneau nerveux à $300 \mu \mathrm{m}$ de l'apex; capsule buccale haute de $7 \mu \mathrm{m}$; œsophage long de $1500 \mu \mathrm{m}$ (portion musculaire de $530 \mu \mathrm{m}$ ) ; vulve à $3550 \mu \mathrm{m}$ de l'apex et portion impaire de l'ovéjecteur longue de $1900 \mu \mathrm{m}$; queue longue de $650 \mu \mathrm{m}$. Crêtes cuticulaires du corps débutant à $8100 \mu \mathrm{m}$ de la tête et se terminant à $1300 \mu \mathrm{m}$ de l'extrémité caudale.

- $\sigma^{A}$ allotype : corps long de $18 \mathrm{~mm}$ et large de $225 \mu \mathrm{m}$; anneau nerveux à $195 \mu \mathrm{m}$ de l'apex; capsule buccale haute de $7 \mu \mathrm{m}$; œsophage long de $1575 \mu \mathrm{m}$; spicule gauche long de $260 \mu \mathrm{m}$ avec manche de $130 \mu \mathrm{m}$; spicule droit long de 


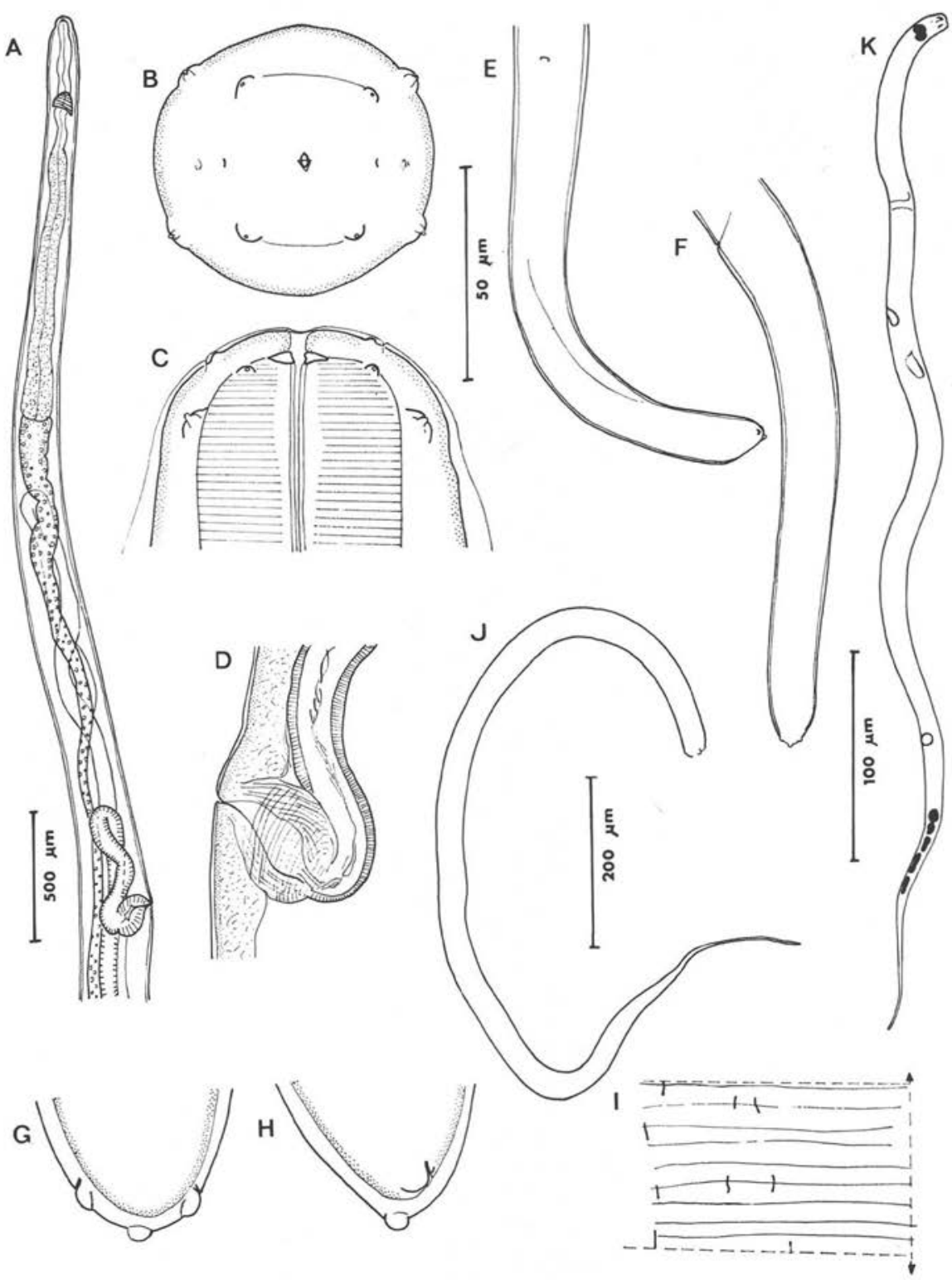

Fig. 1. Breinlia (B.) manningi n. sp., ‥ A : région antérieure, vue latérale; B et C: tête, vues apicale et médiane ; D : vagin et début de l'ovéjecteur; E : queue (anus en vue ventrale) ; $\mathrm{F}$ : idem, vue latérale; $\mathrm{G}$ et $\mathrm{H}$ : extrémité caudale, vues médiane et latérale ; I : ornementation cuticulaire à mi-corps, champ submédian; $\mathrm{J}$ : microfilaire extraite de l'utérus ; $\mathrm{K}$ : microfilaire sanguine sur un frottis. (A, éch. : $500 \mu \mathrm{m}$; B, C, G, H, J et K, éch. : $50 \mu \mathrm{m}$; D, E, F, éch. : $200 \mu \mathrm{m}$; I, éch. : $100 \mu \mathrm{m}$ ). 


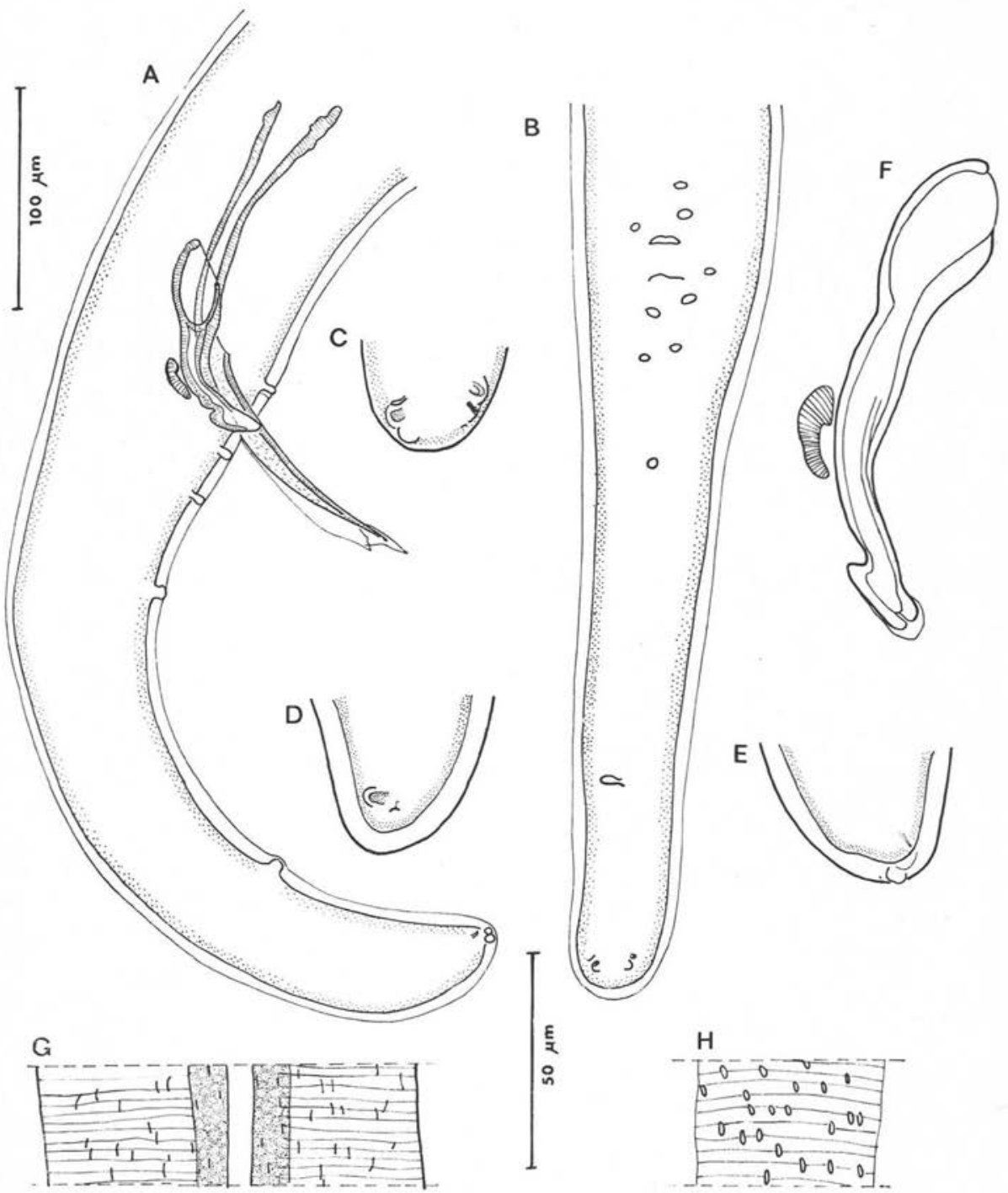

Fig. 2. Breinlia (B.) manningi n. sp., $\sigma^{\star}$. A et B : région caudale, vues latérale et ventrale ; $\mathrm{C}, \mathrm{D}$ et $\mathrm{E}$ : extrémité caudale, vue médiane, latérale gauche et latérale droite; $\mathrm{F}$ : spicule droit et gubernaculum, vue latérale droite; $\mathrm{G}$ et $\mathrm{H}$ : ornementation cuticúlaire du corps, respectivement à mi-corps (vue latérale) et à $1650 \mu \mathrm{m}$ de l'extrémité caudale (champ submédian). (A, B, G, éch. : $100 \mu \mathrm{m}$; C, D, E, F, H, éch. : $50 \mu \mathrm{m}$ ). 
$100 \mu \mathrm{m}$; gubernaculum long de $15 \mu \mathrm{m}$; queue longue de $380 \mu \mathrm{m}$; crêtes cuticulaires du corps commençant à $2500 \mu \mathrm{m}$ de la tête et se terminant à $1100 \mu \mathrm{m}$ de l'extrémité caudale.

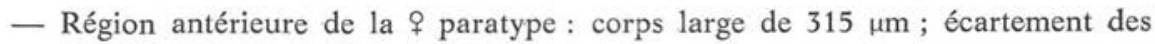
papilles céphaliques de $50 \mu \mathrm{m}$ sur $40 \mu \mathrm{m}$; anneau nerveux et pore excréteur à $310 \mu \mathrm{m}$ et $370 \mu \mathrm{m}$ de l'apex; capsule buccale haute de $8 \mu \mathrm{m}$; œsophage long de $1500 \mu \mathrm{m}$; vulve à $2500 \mu \mathrm{m}$ de l'apex.

- Microfilaires extraites de l'utérus de la $q$ paratype : corps longs de 245, 238, 235 et $250 \mu \mathrm{m}$ sur 5,5 $\mu \mathrm{m}$ de large. Microfilaire sanguine (frottis coloré au Giemsa) : corps long de $235 \mu \mathrm{m}$, large de 5,5 $\mu \mathrm{m}$; espace céphalique long de $6 \mu \mathrm{m}$; anneau nerveux, pore excréteur et noyau excréteur à 48,75 et $85 \mu \mathrm{m}$ de l'apex ; queue longue de $168 \mu \mathrm{m}$; filament caudal long de $32 \mu \mathrm{m}$.

\section{Discussion}

La Filaire du Sciuridae Menetes berdmorei se classe aisément dans le sous-genre Breinlia Yorke et Maplestone, 1926, qui comprend des parasites de Marsupiaux dans la région australienne, de Lémuriens et de Rongeurs dans la région indo-malaise (cf. Chabaud et Bain, 1976).

Quatre espèces sont décrites dans le sud-est asiatique : B. (B.) sergenti (Mathis et Léger, 1909), chez le Lémurien Nycticebus coucang, réétudiée par Petter (1958), $B$. (B.) booliati Singh et Ho, 1973, chez des Muridés et des Sciuridés, dans la péninsule malaise et à Bornéo (Mak et Lim, 1974; Bain, Tibayrenc et Mak, 1979), B. (B.) spratti Bain et coll., 1979, parasite de Sciurinae en Malaisie et $B$. (B.) petauristi Chowattukunnel et Esslinger, 1979, parasite de Sciuridae Petauristinae dans le sud de l'Inde. Ces 4 espèces sont nettement plus grandes que nos spécimens dont elles diffèrent en outre par d'autres caractères: $B$. $(B$.) booliati a une microfilaire plus petite $(188.206 \mu \mathrm{m}$ au lieu de $235-250 \mu \mathrm{m}), B$. (B.) spratti, une microfilaire plus grande $(270-320 \mathrm{~m})$, ainsi qu'un spicule droit à talon peu marqué et un gubernaculum sans entaille.

Les 2 autres espèces ont une microfilaire de même taille que nos spécimens, mais $B$. (B.) petauristi a un spicule droit sans talon et un gubernaculum rond en section transversale et $B$. (B.) sergenti a des papilles caudales beaucoup plus nombreuses, une queue $q$ sans mucron axial terminal et des crêtes cuticulaires qui naissent plus antérieurement (fin de l'œsophage).

A ces 4 espèces, il faut ajouter $B$. (B.) ratti, mentionné chez Rattus jalorensis en Thailande par Viraboonchai et coll. (1974! et dont nous n'avons pas trouvé la description.

Quant aux parasites de Marsupiaux dans la région australienne, ils sont en général beaucoup plus grands que nos spécimens, à l'exception de deux espèces : $B$. $(B$.) 
rarum (Johnston et Mawson, 1938) et B. (B.) dentonensis (Spratt et Varughese, 1975), tous deux parasites de Macropodidés.

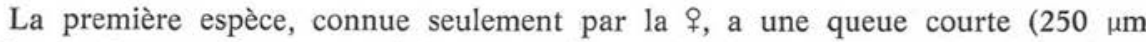
au lieu de $650 \mu \mathrm{m}$ chez nos spécimens) et de grandes languettes caudales latérales.

La deuxième espèce est l'un des deux représentants d'un petit groupe atypique de Breinlia (groupe B, sous-groupe 1 de Spratt et Varughese) caractérisé par la microfilaire sans filament caudal, les papilles toutes groupées en arc de cercle en arrière du cloaque et le gubernaculum très épais; elle s'oppose donc aisément à nos spécimens.

La Filaire de Menetes berdmorei constitue donc une nouvelle espèce que nous nommons Breinlia (B.) manningi n. sp.

\section{Développement larvaire de B. (B.) manningi n. sp.}

\section{chez Aedes spp.}

Aedes aegypti (souche Gkep) et Aedes togoi sont gorgés sur le Menetes filarien. Chez les 2 espèces, le développement s'effectue dans le tissu adipeux en 10 jours à $27^{\circ}$; la mue I a lieu le $6^{\mathrm{e}}$ jour ; dans les 2 cas, un moustique sur 5 ou 6 en moyenne est parasité ( 1 ou 2 larves par moustique).

La morphologie du stade infestant est représentée sur la figure 3 .

Dimensions : un stade I de 5 jours est long de $360 \mu \mathrm{m}$, large de $27 \mu \mathrm{m}$; queue longue de $95 \mu \mathrm{m}$, filament caudal long de $55 \mu \mathrm{m}$.

Une larve en mue I est longue de $335 \mu \mathrm{m}$, large de $40 \mu \mathrm{m}$, avec un filament caudal de $50 \mu \mathrm{m}$.

Un stade infestant $q$ est long de $950 \mu \mathrm{m}$, large de $22 \mu \mathrm{m}$; capsule buccale haute de $7 \mu \mathrm{m}$; anneau nerveux et pore excréteur à 100 et $150 \mu \mathrm{m}$ de l'apex ; œsophage long de $490 \mu \mathrm{m}$ (portion musculaire longue de $175 \mu \mathrm{m}$ ); rectum et queue longs de 55 et $75 \mu \mathrm{m}$; languettes caudales longues de $3,5 \mu \mathrm{m}$; ébauche génitale à $260 \mu \mathrm{m}$ de l'apex.

Un stade infestant $\delta$ est long de $1150 \mu \mathrm{m}$, large de $23 \mu \mathrm{m}$; capsule buccale haute de $7 \mu \mathrm{m}$; anneau nerveux à $115 \mu \mathrm{m}$ de l'apex; œsophage long de $590 \mu \mathrm{m}$ (portion musculaire de $200 \mu \mathrm{m}$ ); queue longue de $80 \mu \mathrm{m}$; ébauche génitale à $25 \mu \mathrm{m}$ de la jonction œsophage-intestin.

Autres stades infestants : corps long de 970 à $1200 \mu \mathrm{m}$, large de 18 à $25 \mu \mathrm{m}$; queue longue de 75 à $92 \mu \mathrm{m}$. 

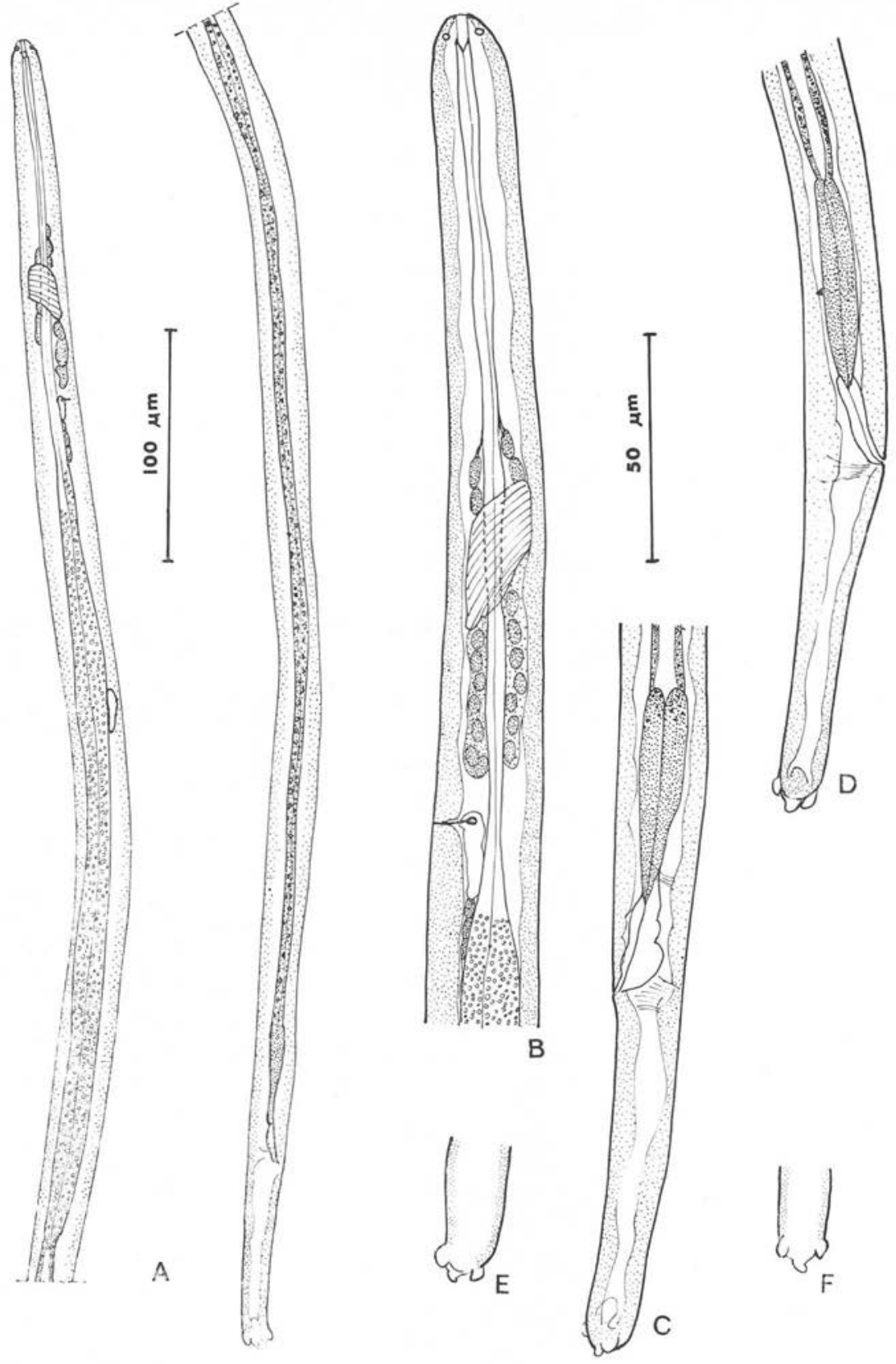

Fig. 3. Breinlia (B.) manningi n. sp., forme infestante. A: aspect général d'une larve (larve en 2 morceaux); B : région antérieure, vue latérale; $C$ et D : régions caudales, vue latérale (les queues sont légèrement spiralées); $\mathrm{E}$ et $\mathrm{F}$ : deux extrémités caudales, vue médiane. (A, éch. : $100 \mu \mathrm{m}$; le reste, éch. : $50 \mu \mathrm{m}$ ). 


\section{Conclusion}

Les Breinlia (Breinlia), bien diversifiés en Australie, apparaissent plus nombreux en Asie qu'on ne le supposait : à la Filaire de Lémurien, B. (B.) sergenti, la plus anciennement connue dans cette région, se sont ajoutées durant les dix dernières années 4 autres espèces, parasites de Sciuridés (Sciurinae et Petauristinae) et de Muridés : B. (B.) booliati, B. (B.) spratti, B. (B.) petauristi et B. (B.) manningi n. sp.

Ces 5 espèces, bien que parasites d'hôtes variés, sont très proches les unes des autres; elles semblent donc s'être formées postérieurement à l'évolution de leurs hôtes, par des captures relativement récentes.

Les 3 cycles de Breinlia connus, tous asiatiques [B. (B.) sergenti, in Zaman et Chellapah, 1968, et Ramachandran et Dunn, 1968 ; B. (B.) booliati in Ho et coll., 1973 ; B. (B.) manningi n. sp., cf. ci-dessus], permettent de dégager les caractères larvaires, biologiques et morphologiques du genre : développement dans le tissu adipeux d'un moustique ; long filament caudal anucléé au stade I (microfilaire et forme en saucisse) ; stades infestants longs d'un peu plus d'un millimètre, ayant un œsophage égal à la moitié du corps, une queue relativement très longue $(68-92 \mathrm{~m})$ *, terminée par une pointe conique (celle-ci est absente chez certains spécimens) et deux languettes latérales, bien saillantes et plus ou moins coniques.

\section{Bibliographie}

Bain O., Tibayrenc M., Mak J.W.: Deux espèces de Breinlia (Filarioidea) chez un Ecureuil en Malaisie. Bull. Mus. Nat. Hist. Nat., Paris, $4^{e}$ sér., 1979, 1, sect. A, 191-197.

Chabaud A.-G., Bain O.: La lignée Dipetalonema. Nouvel essai de classification. Ann. Parasitol. Hum. Comp., 1976, 51, 365-397.

Chowattukunnel J. T., Esslinger J.H. : A new species of Breinlia (Breinlia) (Nematoda : Filarioidea) from the south indian flying squirrel, Petaurista philippensis (Elliot). J. Parasitol., 1979, 65, 375-378.

Ho B.C., Singh M., Lim B. L. : Observations on the development of a new filaria (Breinlia booliati Singh and Ho, 1973) of a rat Rattus sabanus in the mosquitoe Aedes togoi. J. Helm., 1973, 47, 135-140.

Johnston T.H., Mawson P. M. : An account of some filarial parasites of australian Marsupials. Trans. R. Soc. South Aust., 1938, 62, 107-121.

Mak J.W., Lim B. L.: New host of Breinlia booliati in wild rats from Sarawak with further observations on its morphology. S.E. Asian J. Trop. Med. Pub. Hlth., 1974, 5, 22-30.

Manning G.S., Harrison B. A., Wooding W. L., Subhakul M. : Studies on Brugia tupaiae in Thailand. Ann. trop. Med. Parasit., 1972, 66, 497-503.

* Les mensurations de B. (B.) booliati sont prises au laboratoire sur des larves récoltées chez Aedes togoi, infestés par une souche issue de Rattus sabanus, n'ayant subi qu'un seul passage sur Rat blanc. 
Petter A.-J. : Filaria sergenti Mathis et Léger, 1909, parasite d'un Nycticèbe (Nycticebus tardigradus Link), appartient au genre Breinlia Yorke et Maplestone, 1926. Bull. Soc. Zool. Fr., 1958, 83, 423-429.

Ramachandran C. P., Dunn F. L.: The development of Breinlia sergenti (Dipetalonematidae) in Aedes mosquitoes. Ann. trop. Med. Parasit., 1968, 62, 441-454.

Singh M., Ho B. C. : Breinlia booliati sp. n. (Filarioidea: Onchocercidae), a Filaria of the malayan forest rat, Rattus sabanus (Thos). J. Helm., 1973, 47, 127-133.

Spratt D. M., Varughese G. : A taxonomic revision of filarioid Nematodes from autralian Marsupials. Austr. J. Zool., suppl. ser., 1975, n 35, 1-99.

Viraboonchai S., Guptavnij P., Sucharit S., Harinasuta C.: Breinlia ratti in natural host (Rattus jalorensis) and experimental hosts. S.E. Asian J. Trop. Med. Pub. Hlth., 1974, 5, 143.

Walker J. C., Mc Millan B.: Breinlia mackerrasae n. sp. (Nematoda: Filarioidea) from the peramelid marsupial, Isoodon macrourus, with a note on Breinlia sp. from the " mumut » Peramelidae. J. Helm., 1974, 48, 39-45.

Zaman V., Chellapah W. T. : Studies on vector susceptibility and larval morphology of a Filaria of the slow loris, Nycticebus coucang. Ann. Trop. Med. Parasit., 1968, 62, 450-454. 